\title{
On the generalized eigenvalue problem for the Rossby wave vertical velocity in the presence of mean flow and topography
}

Article

Published Version

Tailleux, R. (2012) On the generalized eigenvalue problem for the Rossby wave vertical velocity in the presence of mean flow and topography. Journal of Physical Oceanography, 42 (6). pp. 1045-1050. ISSN 0022-3670 doi: https://doi.org/10.1175/JPOD-12-010.1 Available at https://centaur.reading.ac.uk/34401/

It is advisable to refer to the publisher's version if you intend to cite from the work. See Guidance on citing.

To link to this article DOI: http://dx.doi.org/10.1175/JPO-D-12-010.1

Publisher: American Meteorological Society

Publisher statement: Publisher retains copyright

All outputs in CentAUR are protected by Intellectual Property Rights law, including copyright law. Copyright and IPR is retained by the creators or other copyright holders. Terms and conditions for use of this material are defined in the End User Agreement.

$\underline{\text { www.reading.ac.uk/centaur }}$

\section{CentAUR}


Central Archive at the University of Reading

Reading's research outputs online 


\title{
On the Generalized Eigenvalue Problem for the Rossby Wave Vertical Velocity in the Presence of Mean Flow and Topography
}

\author{
RÉMI TAILLEUX \\ Department of Meteorology, University of Reading, Reading, United Kingdom
}

(Manuscript received 12 January 2012, in final form 4 April 2012)

\begin{abstract}
In a series of papers, Killworth and Blundell have proposed to study the effects of a background mean flow and topography on Rossby wave propagation by means of a generalized eigenvalue problem formulated in terms of the vertical velocity, obtained from a linearization of the primitive equations of motion. However, it has been known for a number of years that this eigenvalue problem contains an error, which Killworth was prevented from correcting himself by his unfortunate passing and whose correction is therefore taken up in this note. Here, the author shows in the context of quasigeostrophic (QG) theory that the error can ultimately be traced to the fact that the eigenvalue problem for the vertical velocity is fundamentally a nonlinear one (the eigenvalue appears both in the numerator and denominator), unlike that for the pressure. The reason that this nonlinear term is lacking in the Killworth and Blundell theory comes from neglecting the depth dependence of a depth-dependent term. This nonlinear term is shown on idealized examples to alter significantly the Rossby wave dispersion relation in the high-wavenumber regime but is otherwise irrelevant in the long-wave limit, in which case the eigenvalue problems for the vertical velocity and pressure are both linear. In the general dispersive case, however, one should first solve the generalized eigenvalue problem for the pressure vertical structure and, if needed, diagnose the vertical velocity vertical structure from the latter.
\end{abstract}

\section{Introduction}

A central question in the theory of ocean variability is how the barotropic and baroclinic normal modes of the standard linear theory (SLT) (e.g., Gill 1982) are modified by the presence of a background mean flow and variable topography. To address this issue, progress over the past decades has principally come from investigating the nature of the solutions of the equations of motion linearized around a background mean flow, most often in the context of quasigeostrophic (QG) theory. Under the Wentzel-Kramers-Brillouin approximation, which assumes that the scales over which the background mean flow and topography vary are large compared to that of the waves considered, approximately separable wave solutions still exist, whose vertical structure can be obtained as the eigenmodes of a nonself-adjoint eigenvalue problem. When the problem is formulated by

Corresponding author address: Rémi Tailleux, Department of Meteorology, University of Reading, Earley Gate, P.O. Box 243, Reading RG6 6BB, United Kingdom.

E-mail: r.g.j.tailleux@reading.ac.uk linearizing the quasigeostrophic potential vorticity (PV) evolution equation around a background zonal mean flow, for instance, the generalized eigenvalue problem thus obtained is generally naturally formulated in terms of the vertical structure for the pressure [e.g., see Fu and Chelton (2001) and Aoki et al. (2009) for recent examples].

In the classical SLT, that is, in the absence of mean flow and topography, it has long been known that the eigenvalue problem defining the standard barotropic and baroclinic modes can be indifferently formulated in terms of the vertical structure for either pressure or vertical velocity. Yet, we were unable to find any published derivation of the generalized eigenvalue problem (i.e., accounting for mean flow and topography) for the vertical structure of the vertical velocity in the context of QG theory. Motivated by the fact that boundary conditions are generally simpler for the vertical velocity, Killworth and Blundell (Killworth and Blundell 2004, hereafter KB04; Killworth and Blundell 2005, hereafter KB05; Killworth and Blundell 2007, hereafter KB07) sought to formulate a generalized eigenvalue problem for the vertical structure of the vertical velocity from 
directly linearizing the primitive equations. Using QG scaling, they first obtained linearized equations for the horizontal velocity components in terms of the Welander (1959) $M$ function, which once inserted into the continuity equation led to the following expression for the vertical derivative of $w$ :

$w_{z}=\frac{i k M_{z}}{2 \Omega a^{2} \sin ^{2} \theta}-\frac{i R M_{z}}{a^{2} f^{2}}\left(\frac{k^{2}}{\cos ^{2} \theta}+l^{2}\right)+$ small,

where $R=\mathbf{k}_{\mathrm{dim}} \cdot \overline{\mathbf{u}}-\omega$ is minus the Doppler-shifted frequency $\omega ; \bar{u}$ is the zonal background mean flow; $\mathbf{k}_{\mathrm{dim}}=$ $\left(k_{x}, k_{y}\right)$ is the dimensional wave vector; $(k, l)$ are the angular zonal and meridional wavenumbers, which are related to the dimensional wavenumbers $\left(k_{x}, k_{y}\right)$ by $k_{x}=$ $k /(a \cos \theta)$ and $l=k_{y} / a ; a$ is the earth's radius; $\theta$ is the latitude; $\Omega$ is the earth's rotation rate; and $f=2 \Omega \sin \theta$ is the local Coriolis parameter. At this point, KB04 sought to derive an expression for $w$ by vertically integrating Eq. (1), which they took to be given by

$$
w=\frac{i k M}{2 \Omega a^{2} \sin ^{2} \theta}-\frac{i R M}{a^{2} f^{2}}\left(\frac{k^{2}}{\cos ^{2} \theta}+l^{2}\right) .
$$

Such a derivation is valid, however, only if $R$ can be assumed to be independent of $z$ but, as noted earlier, $R$ is given by

$$
R=\mathbf{k}_{\operatorname{dim}} \cdot \overline{\mathbf{u}}-\omega
$$

and in general will depend on $z$ because the background mean flow depends on $z$.

The above error was first identified by R. Samelson (2007, personal communication), who had pointed it out to Peter Killworth at the time. However, despite his best efforts, Peter Killworth passed away before he could find a cure to the problem. The main objective of this paper is twofold: 1) to clarify the nature of the error and show how to redress it and 2) to understand how the error affects some of KB04's conclusions regarding the nature of the dispersion relation of Rossby waves in the presence of a background mean flow and topography. The issue is important to clarify because it also affects KB05's results and KB07's discussion of forced modes and baroclinic instability and is expected to alter some of the conclusions of the comparison of the KB04 theoretical dispersion relations against observations recently carried out by Maharaj et al. (2007, 2009).

In this paper, we note that the KB04 theory appears to rely on the same scaling arguments as those underlying the construction of QG theory and, hence, seek to derive a generalized eigenvalue problem for the vertical velocity directly from QG theory, which is done in section 2. The main result is that the eigenproblem thus obtained only differ from that of KB04 by a term that makes the QG eigenvalue problem for the vertical velocity nonlinear and hence intractable. Section 3 illustrates on some idealized examples that such a term affects the KB04 dispersion relations mostly in the high wavenumbers regime. Section 4 summarizes the results and discusses some of its consequences.

\section{Generalized eigenvalue problem for $w$ in QG theory}

The standard starting point for generalizing the SLT to account for the effects of a background mean flow and topography is the QG evolution equation for the potential vorticity equation,

$$
\frac{D_{g} q}{D t}=0
$$

where $D_{g} / D t=\partial_{t}+J(\Psi, \cdot)$ is advection by the geostrophic velocity $\left(u_{g}, v_{g}\right)=-\Psi_{y}, \Psi_{x}$, with $\Psi$ being the geostrophic streamfunction, and $q$ is the potential vorticity,

$$
q=f_{0}+\beta y+\frac{\partial^{2} \Psi}{\partial x^{2}}+\frac{\partial^{2} \Psi}{\partial y^{2}}+\frac{\partial}{\partial z}\left(\frac{f_{0}^{2}}{N^{2}} \frac{\partial \Psi}{\partial z}\right) .
$$

The geostrophic streamfunction $\Psi$ is related to the buoyancy $b$, pressure $p$, and vertical velocity $w$ through the following relations:

$$
\begin{aligned}
& b=f_{0} \frac{\partial \Psi}{\partial z}, \quad p=\rho_{0} f_{0} \Psi \\
& w=-\frac{D_{g}}{D t}\left(\frac{f_{0}}{N^{2}} \frac{\partial \Psi}{\partial z}\right)
\end{aligned}
$$

(e.g., Vallis 2007). As shown by several authors (e.g., Fu and Chelton 2001; Aoki et al. 2009), the QG evolution equation linearized around a zonal background mean flow $\bar{u}=\bar{u}(z)$ (neglecting $\bar{u}_{y y}$ relative to $\beta$ ) admits separable wavelike solutions $\Psi \propto F(z) e^{i\left(k_{x} x+k_{y} y-\omega t\right)}$, whose vertical structure $F$ can be regarded as the eigenmodes of the eigenvalue problem:

$$
(\bar{u}-c)\left[\frac{d}{d z}\left(\frac{f_{0}^{2}}{N^{2}} \frac{d F}{d z}\right)-K^{2} F\right]+\left[\beta-\frac{d}{d z}\left(\frac{f_{0}^{2}}{N^{2}} \frac{d \bar{u}}{d z}\right)\right] F=0
$$

where $K^{2}=k_{x}^{2}+k_{y}^{2}$, with suitable boundary conditions whose precise form depends on whether a variable or 
flat bottom topography is considered, and $c=\omega / k_{x}$ is the zonal phase speed.

Our objective is to obtain the corresponding eigenvalue problem for the vertical velocity $w$. To that end, linearizing Eq. (7) yields the following linearized expression for $w$ :

$$
w=-\left(\frac{\partial}{\partial t}+\bar{u} \frac{\partial}{\partial x}\right) \frac{f_{0}}{N^{2}} \frac{\partial \Psi^{\prime}}{\partial z}+\frac{f_{0}}{N^{2}} \frac{d \bar{u}}{d z} \frac{\partial \Psi^{\prime}}{\partial x} .
$$

This implies for the vertical structure of wavelike solutions $w=W(z) e^{i\left(k_{x} x+k_{y} y-\omega t\right)}$ that it is related to the pressure vertical structure $F$ through

$$
W=-i f_{0} k_{x}\left[\frac{(\bar{u}-c)}{N^{2}} \frac{d F}{d z}-\frac{1}{N^{2}} \frac{d \bar{u}}{d z} F\right] .
$$

To arrive at the eigenvalue problem satisfied by $W$, we successively differentiate the latter expression with respect to $z$ by making use of the links between $F$ and $W$ to successively eliminate terms involving $F$ and its derivatives. Thus, differentiating the latter equation a first time yields

$$
\frac{d W}{d z}=-i f_{0} k_{x}\left[(\bar{u}-c) \frac{d}{d z}\left(\frac{1}{N^{2}} \frac{d F}{d z}\right)-\frac{d}{d z}\left(\frac{1}{N^{2}} \frac{d \bar{u}}{d z}\right) F\right]
$$

By taking advantage of the fact that $F$ satisfies the eigenvalue problem (8), it is possible to remove the second-order term in $F$ to simplify this expression as follows:

$$
\frac{d W}{d z}=-\frac{i k_{x}}{f_{0}}\left[(\bar{u}-c) K^{2}-\beta\right] F=\frac{i k_{x}}{f_{0}}\left[\beta-K^{2}(\bar{u}-c)\right] F
$$

in which all first and second derivatives in $F$ have been eliminated. Taking the vertical derivative a second time yields, this time,

$$
\frac{d^{2} W}{d z^{2}}=\frac{i k_{x}}{f_{0}}\left[\beta-K^{2}(\bar{u}-c)\right] \frac{d F}{d z}-\frac{i k_{x} K^{2}}{f_{0}} \frac{d \bar{u}}{d z} F .
$$

By using Eq. (10) it is possible to express $d F / d z$ in terms of $F$ and $W$ as

$$
\frac{d F}{d z}=\frac{1}{\bar{u}-c} \frac{\partial \bar{u}}{\partial z} F+\frac{i N^{2}}{f_{0} k_{x}(\bar{u}-c)} W,
$$

and then, by using Eq. (12), it is possible to express $F$ in terms of $d W / d z$. As a result, the following eigenproblem is obtained:

$$
\begin{gathered}
(\bar{u}-c) \frac{d^{2} W}{d z^{2}}-\left[1-\frac{K^{2}(\bar{u}-c)}{\beta-K^{2}(\bar{u}-c)}\right] \frac{d \bar{u}}{d z} \frac{d W}{d z} \\
+\frac{\left[\beta-K^{2}(\bar{u}-c)\right] N^{2}}{f_{0}^{2}} W=0 .
\end{gathered}
$$

The surprising result here is that Eq. (15) is a nonlinear eigenvalue problem because of the term $K^{2}(\bar{u}-c) /$ $\left[\beta-K^{2}(\bar{u}-c)\right]$, which involves the eigenvalue $c$ both in the numerator and denominator. In contrast, the eigenvalue problem derived by KB04 (in absence of meridional mean flow) is given by

$$
\begin{aligned}
(\bar{u}-c) \frac{d^{2} W_{\mathrm{KB}}}{d z^{2}}-\frac{d \bar{u}}{d z} \frac{d W_{\mathrm{KB}}}{d z} & \\
+ & \frac{\left[\beta-(\bar{u}-c) K^{2}\right] N^{2}}{f_{0}^{2}} W_{\mathrm{KB}}=0 .
\end{aligned}
$$

The two eigenproblems are identical but for the missing nonlinear term in Eq. (16). In the long-wave limit studied by many authors (e.g., Killworth et al. 1997; Tailleux 2004; de Verdière and Tailleux 2005), however, the nonlinear term vanishes and the eigenvalue problems for the pressure and vertical velocity are equally simple and linear. In some other instances, such as in the case of internal waves in the traditional $f$-plane approximation, it is in terms of the vertical velocity that the problem is most conveniently formulated as it is the problem in terms of pressure that becomes nonlinear [e.g., Gill 1982, Eq. (8.4.10)].

\section{Particular example of the differences}

As mentioned above, the nonlinear term $K^{2}(\bar{u}-c) /$ $\left[\beta-K^{2}(\bar{u}-c)\right]$ responsible for the difference between the two eigenproblems given by Eqs. (15) and (16) clearly vanishes in the long-wave limit $K \rightarrow 0$ so that we expect it to affect the eigensolutions only at large wavenumbers. That this is indeed the case is illustrated here in the particular case of the following idealized mean flow and stratification:

$$
\begin{aligned}
\bar{u}(z) & =u_{\min }+\Delta u_{0} \exp \left[\left(z-z_{0}\right)^{2} / \delta^{2}\right] \\
N^{2}(z) & =N_{0}+\Delta N \exp \left[\left(z-z_{0}\right)^{2} / \delta^{2}\right],
\end{aligned}
$$

which are displayed in Fig. 1. The consideration of an idealized example is sufficient for the present purposes of illustrating that the error made in the Killworth and Blundell (KB) papers is not innocuous. A more complete investigation of the consequences of the error made in KB04 on the conclusions of all the papers that rely on 


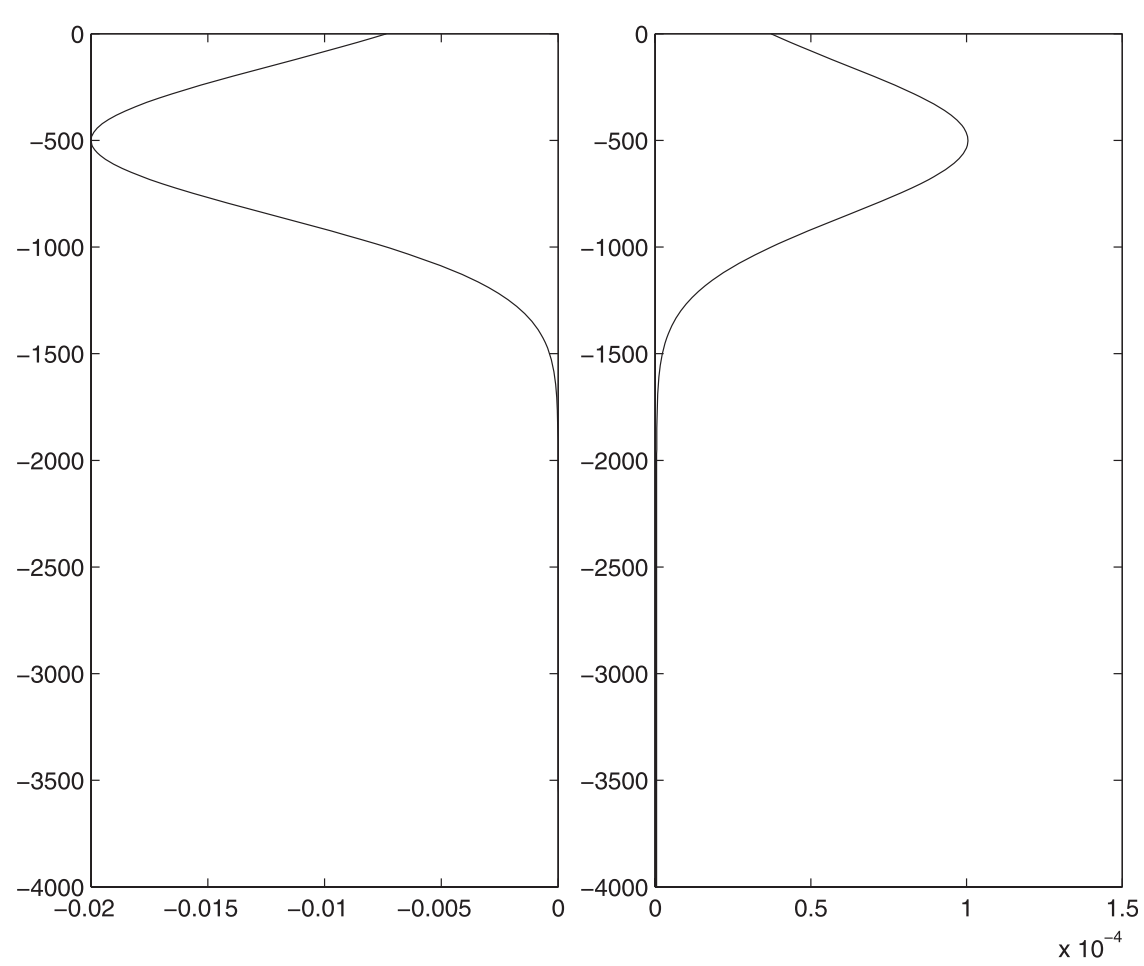

FIG. 1. Idealized profiles for (right) the squared buoyancy frequency $N^{2}\left(\mathrm{~s}^{-1}\right)$ and (left) zonal velocity profile $\left(\mathrm{m} \mathrm{s}^{-1}\right)$.

KB's papers is beyond the scope of this paper. The dispersion relations for the $\mathrm{QG}$ and $\mathrm{KB}$ eigenproblems were computed by solving the discretized versions of the QG eigenvalue problem for pressure and the KB04 eigenproblem for the vertical velocity by using the Matlab standard eigenvalue routines. To compare the vertical velocity in each theory, the vertical velocity modal structure was diagnosed using the pressure modal structure by using the discretized version of Eq. (10).

In the absence of a background mean flow, both QG and KB theories should be strictly equivalent. This is found to be the case, as illustrated in the left panels of Figs. 2 and 3, corresponding respectively to use of the standard flat-bottom boundary condition and that of bottom-pressure compensation theory of Tailleux and McWilliams (2001), which can be regarded as a limiting case of the effect of bottom topography in the infinitely steep slope limit (e.g., Tailleux 2003). As discussed above, we expect the two theories to yield dissimilar results in the presence of mean flow primarily at large wavenumbers. This is illustrated in the right panels of Figs. 2 and 3, which show that, for wavenumbers larger than the Rossby radius of deformation, the two theories may start to differ dramatically, demonstrating the importance of the corrective term overlooked by KB04 in such a region of the wavenumber space, both for a flat bottom and the bottom-pressure compensation boundary conditions. Note that, in the asymptotic limit $k_{x} \rightarrow-\infty$, the dispersion relationship becomes quasi nondispersive and given by $\omega=-u_{\min } k_{x}$, where $u_{\min }$ is the absolute minimum value of $\bar{u}(z)$ in the vertical, as demonstrated by Gnevyshev and Shrira (1989). Although the KB dispersion relationship also appears to be quasi nondispersive at high wavenumbers, its behavior appears nevertheless quite different from that of the QG case. Moreover, we failed to obtain an analytical result for the asymptotic behavior of the KB dispersion relationship for $k_{x} \rightarrow-\infty$.

\section{Summary and conclusions}

In this paper, we derived the generalized QG eigenvalue problem for the vertical velocity normal mode structure of Rossby waves in presence of a background mean flow and topography. Previously, such an eigenproblem had been formulated only for the pressure. Surprisingly, the vertical velocity eigenproblem appears to be nonlinear (the eigenvalue appears both in the numerator and denominator), which is very uncommon and not easily anticipated given that the eigenproblem for the pressure is linear. Such a result shows how the 

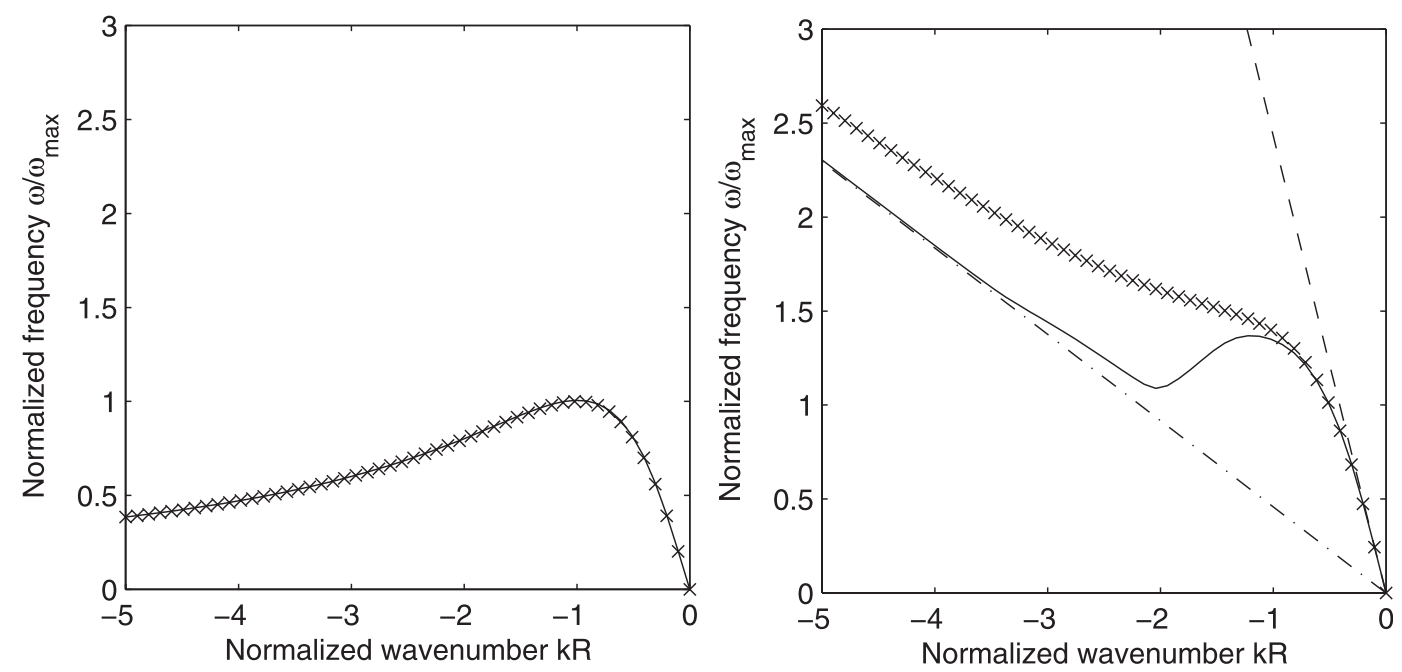

FIG. 2. Comparison of QG (solid line) and the KB (crosses) flat-bottom theories. (left) Dispersion relation in absence of mean flow as predicted by the classical QG theory (solid line) and the KB theory (crosses). (right) Dispersion relation for Rossby waves affected by the idealized Gaussian mean flow illustrated in Fig. 1, as predicted by the classical QG theory (continuous line) and KB theory (crosses). The dashed-dotted line represents the nondispersive dispersion relationship $\omega=u_{\min } k_{x}$; the dashed line represents the nondispersive relationship tangent at $k_{x}=0$ : that is, $\omega=c\left(k_{x}=0\right) k$, where $u_{\min }$ is the absolute minimum of the horizontal zonal velocity along the vertical (which is strictly negative and located at $z=-500 \mathrm{~m}$, according to Fig. 1). Frequency is normalized by the maximum frequency of the flat-bottom, no-mean-flow standard linear theory; the zonal wavenumber is normalized by the inverse of the Rossby radius of deformation.

KB04 derivation might be corrected; at the same time, it also shows that the actual eigenproblem for the vertical velocity is not easily tractable and that the investigation of the propagation properties of Rossby waves in the presence of mean flow and topography is more easily addressed by solving the pressure eigenvalue problem. Whenever the vertical velocity structure $W$ is needed, it is most conveniently diagnosed a posteriori from the
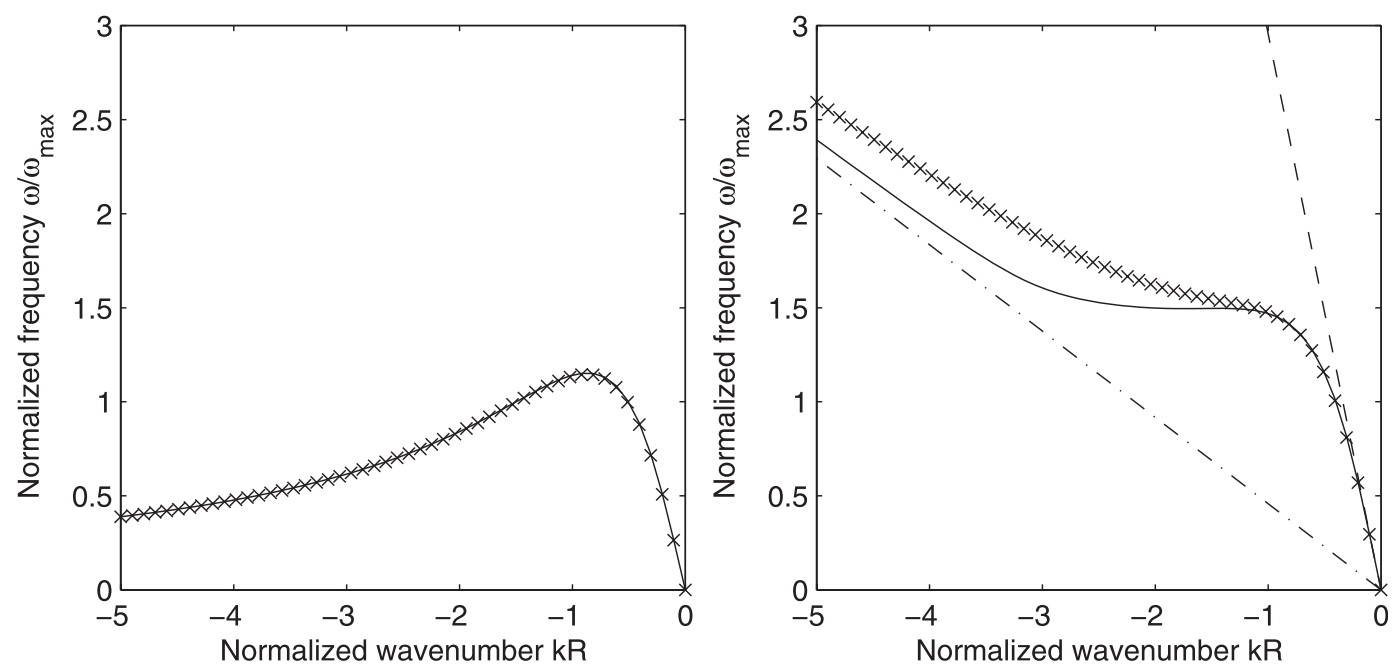

FIG. 3. Comparison of the QG (solid line) and KB (crosses) theories using the bottom boundary condition of the bottom-pressure compensation theory of Tailleux and McWilliams (2001). (left) Dispersion relation in absence of mean flow as predicted by the classical QG theory (solid line) and KB theory (crosses). (right) Dispersion relation for Rossby waves affected by the idealized Gaussian mean flow illustrated in Fig. 1 as predicted by the classical QG theory (continuous line) and KB theory (crosses). The dashed-dotted line represents the nondispersive dispersion relationship $\omega=u_{\min } k_{x}$, as in Fig. 2; the dashed line represents the nondispersive relationship tangent at $k_{x}=0$ : that is, $\omega=c\left(k_{x}=0\right) k_{x}$. Frequency is normalized by the maximum frequency of the flat-bottom, no-mean-flow theory; the zonal wavenumber is normalized by the inverse of the Rossby radius of deformation. 
knowledge of the pressure vertical structure $F$ by using (the discretized version of) Eq. (10).

The results also show that the error made by KB04 is, in fact, equivalent to neglecting the term making the QG eigenproblem for the vertical velocity nonlinear. Such a term is small in the long-wave limit $K \rightarrow 0$ so that the error is mostly of consequence for understanding the behavior of the Rossby wave dispersion relation at high wavenumbers. The study of the latter regime is an old problem, which was investigated in significant detail by Gnevyshev and Shrira (1989) and is therefore not discussed in more detail here.

Acknowledgments. The author gratefully acknowledges financial support from CNES and Dudley Chelton and Roger Samelson for encouragements in pursuing this study. This paper is dedicated to the memory of Peter Killworth, who was a constant source of inspiration in the study of oceanic Rossby waves. We hope that he would have agreed on the solution proposed.

\section{REFERENCES}

Aoki, K., A. Kubokawa, H. Sasaki, and Y. Sasai, 2009: Midlatitude baroclinic Rossby waves in a high-resolution OGCM simulation. J. Phys. Oceanogr., 39, 2264-2279.

de Verdière, A. C., and R. Tailleux, 2005: The interaction of a baroclinic can flow with long Rossby waves. J. Phys. Oceanogr., 35, 865-879.

Fu, L.-L., and D. B. Chelton, 2001: Large-scale ocean circulation. Satellite Altimetry and Earth Sciences: A Handbook of Techniques and Applications, L.-L. Fu and A. Cazenave, Eds., International Geophysics Series, Vol. 69, Academic Press, 133-169.
Gill, A. E., 1982: Atmosphere-Ocean Dynamics. Academic Press, $662 \mathrm{pp}$.

Gnevyshev, V., and V. Shrira, 1989: Monochromatic Rossby wave transformation in zonal flow critical layer. Izv. Acad. Sci. USSR, Atmos. Oceanic Phys., 25, 628-635.

Killworth, P. D., and J. R. Blundell, 2004: The dispersion relation for planetary waves in the presence of mean flow and topography. Part I: Analytical theory and one-dimensional examples. J. Phys. Oceanogr., 34, 2692-2711.

_ and _ - 2005: The dispersion relation for planetary waves in the presence of mean flow and topography. Part II: Twodimensional examples and global results. J. Phys. Oceanogr., 35, 2110-2133.

— and instability in the presence of mean flow and topography. J. Phys. Oceanogr., 37, 1297-1320.

_ D. B. Chelton, and R. de Szoeke, 1997: The speed of observed and theoretical long extratropical planetary waves. J. Phys. Oceanogr., 27, 1946-1966.

Maharaj, A. M., P. Cipollini, N. J. Holbrook, P. D. Killworth, and J. R. Blundell, 2007: An evaluation of the classical and extended Rossby wave theories in explaining spectral estimates of the first few baroclinic modes in the South Pacific Ocean. Ocean Dyn., 57, 173-187, doi:10.1007/s10236-006-0099-5.

— N. J. Holbrook, and P. Cipollini, 2009: Multiple westward propagating signals in South Pacific sea level anomalies. J. Geophys. Res., 114, C12016, doi:10.1029/2008JC004799.

Tailleux, R., 2003: Comments on "The effect of bottom topography on the speed of long extratropical planetary waves." J. Phys. Oceanogr., 33, 1536-1541.

_ 2004: A WKB analysis of the surface signature and vertical structure of long extratropical baroclinic Rossby waves over topography. Ocean Modell., 6, 191-219.

_ coupling on the speed of extratropical, baroclinic Rossby waves. J. Phys. Oceanogr., 31, 1461-1476.

Vallis, G., 2007: Atmosphere Ocean Circulation. Cambridge University Press, $700 \mathrm{pp}$.

Welander, P., 1959: An advective model of the ocean thermocline. Tellus, 11, 309-318. 